$\mathbf{m} / \mathbf{S}$

médecine/sciences $1985 ; 1: 402-3$

\title{
médecine/sciences mode d'emploi
}

La rédaction

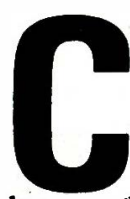

ctte revue internationale est née voici à peine un an, résultat tangible du travail de deux petites équipes de rédaction, et du concours d'auteurs qui ont accepté de relever une sorte de défi : publier des articles de haut niveau scientifique en languc française. Pour que cette ambition ne soit pas présomptueuse et qu'elle corresponde à un besoin réel, nous avons choisi de publier principalement d'excellentes synthèses sur les sujets les plus actuels.

L'époque que nous vivons dans le domaine de la biologie est extraordinaire, et nul parmi les médecins et les chercheurs ne peut se tenir délibérément à l'écart de ce mouvement sans abdiquer en même temps toute ambition de comprendre mieux la vie... et la maladie.

Certes, la progression des connaissances, des moyens d'investigation et des thérapeutiques était déjà rapide avant les années soixante-dix, marquée notamment par la découverte du code génétique, les progrès en immunologie, biologie cellulaire, chimie de synthèse et pharmacologie et la sophistication croissante des moyens d'exploration des organes. C'est bien cependant le développement, à partir de 1976, de l'ensemble des techniques des recombinants d'ADN, encore appelé "génie génétique " qui constitue l'élément déterminant de l'explosion des découvertes actuelles. Directement ou indirectement, tous les processus du vivant sont gouvernés par le code génétique et la maîtrise de ce dernier ouvre tout grand l'accès à des domaines qui se situaient auparavant en dehors du champ de l'expérimentation. La biologie est profondément une science expérimentale et le génie génétique est avant tout un merveilleux outil qui a été utilisé pour éclairer de nouveaux concepts.

Grâce à lui sont tombées les barrières interdisant l'analyse des mécanismes du cancer, de la synthèse spécifique des anticorps et du développement embryonnaire. La neurobiologie et la neuroendocrinologie ont bénéficié de la découverte des gènes codant pour des neuropeptides inconnus. Le "terrain génétique " prédisposant à des maladies aussi fréquentes que l'athérosclérose ou le diabète a maintenant une base moléculaire. Le prix Nobel 
de médecine I 985 vient d'ailleurs de récompenser les remarquables travaux de Joseph $L$. Goldstein et Michael S. Brown sur les mécanismes moléculaires de l'hypercholestérolémie familiale. Cette équipe a découvert les causes de la maladie, a cloné les gènes mutés chez les patients et a pu par conséquent décrypter l'anomalie génétique. Les perspectives de détection précoce des maladies héréditaires, voire de leur traitement par greffe de gènes, se précisent. La vaccination de centaines de millions d'individus contre le paludisme et l'hépatite virale est probablement pour bientôt... grâce à la production de protéines vaccinales par recombinaison génétique. Enfin, c'est dans le contexte de cette époque qu'il a été possible en cinq ans de décrire une nouvelle maladie (le SIDA), d'isoler son agent infectieux et d'analyser son génome, d'espérer ainsi la préparation d'un vaccin. Dans le même temps, les techniques de fécondation in vitro et de conservation d'embryons augmentaient considérablement la maîtrise de l'homme sur sa fécondité et sa reproduction.

Il fallait nous semble-t-il qu'à ce bouillonnement scientifique correspondît un lieu de communication entre chercheurs en biologie, biochimistes, physiologistes et cliniciens. Il apparaît en effet évident que les clivages entre eux sont devenus artificiels : pouvoir détecter le génome du virus de l'hépatite $B$ dans l'ADN d'hépatocytes et, peutêtre, prévoir le développement ultérieur d'un hépatocarcinome... c'est de l'hépatologie; détecter des cellules ou des anticorps qui seront responsables d'un diabète, caractériser le terrain génétique propice à cette maladie en analysant le polymorphisme des gènes HLA... c'est de la diabétologie. Et l'on pourrait multiplier ainsi les exemples de cette nécessaire homogénisation des con- cepts et du langage scientifique. médecine/sciences a choisi de favoriser ce rapprochement entre cliniciens et chercheurs en proposant une formule originale qui comprend des mises au point conceptuelles, des nouvelles d'actualité, des notes originales, des débats ainsi que des articles plus complets élaborant une synthèse sur un sujet et permettant de servir de référence pédagogique ou de base de départ pour des travaux de recherche. Certes, le niveau de dialogue choisi réserve la lecture de médecine/sciences à un public de professionnels, mais nous pensons que l'objectif que nous nous étions assigné excluait toute facilité. La disponibilité d'une subvention temporaire d'agences gouvernementales françaises et québecoises nous imposait par ailleurs de répondre d'abord à l'attente des chercheurs et des scientifiques de chaque côté de l'Atlantique. Notre fierté c'est d'avoir su conquérir, en plus d'abonnés francophones internationaux, l'attention d'un public anglo-saxon.

Il nous reste à donner à notre revue un avenir durable. Seul un nombre plus élevé d'abonnés nous permettra d'atteindre un équilibre financier et de continuer à nous assurer la collaboration d'auteurs de grande qualité scientifique. Votre abonnement individuel et votre aide pour mieux faire connaître notre revue, constituent des manifestations concrètes et nécessaires de votre soutien. Pour mieux répondre à votre attente, nous publierons dans quelques mois un questionnaire qui nous permettra de connaître votre sentiment sur médecine/sciences ainsi que vos suggestions. Nous espérons ainsi qu'ensemble il nous sera possible de développer et d'améliorer notre revue, votre revue, réponse francophone à l'exigence d'une époque de grands bouleversements scientifiques 